\title{
An unusual case of intrarenal coiled and ruptured guidewire
}

\author{
Francesca Manassero $^{1}$, Simona Ortori ${ }^{2}$, Cristina Gabellieri ${ }^{1}$, Michela Gabelloni ${ }^{2}$, Cesare Selli ${ }^{1}$ \\ ${ }^{1}$ Section of Urology and ${ }^{2}$ Section of Radiology, Department of Translational Research, University of Pisa, Italy.
}

\begin{abstract}
Summary Objective. To the best of our knowledge there are only 3 reports of fractured guidewires inside the pelvicalyceal system, successfully removed with endourology techniques, and this is the first one presenting a tightly coiled intraparenchymal section. Material and methods: A 59-year-old woman was hospitalized for surgical treatment of a right kidney of reduced size. Past history revealed pyelolithotomy for a staghorn stone 14 months earlier at another Institution with subsequent ureteral obstruction, one failed attempt at ureteral double-J catheter insertion and one failed attempt at percutaneous nephrostomy placement 5 months postoperatively. Another nephrostomy was placed, but left indwelling briefly. CT scan demonstrated a small-size kidney with residual stone fragments and presence of $a$ "device" in the lower pole. The tapered distal extremity of an hydrophilic guidewire, with a tightly coiled central section wedged in the renal tissue was found inside the nephrectomy specimen.

Results: While the Radiologist who read the CT scan hypothesized that the "device" was a fragment of double-J ureteral stent or nephrostomy catheter, it consisted of the hydrophilic extremity of a guidewire, broken during a previous attempt at nephostomy placement. Perirenal fibrosis and inappropriate angle between the needle and the lower calyx are the likely causes of guidewire coiling during its advancement and subsequent rupture during withdrawal. Conclusions. Urologists must be aware that, although percutaneous nephrostomy has a very high technical success rate, unusual complications like guidewire fracture may occur, and that modern imaging techniques can provide an accurate picture of this condition.
\end{abstract}

KEY WORDS: Nephrostomy; Percutaneous; Radiology; Interventional; Computed Tomography Spiral; Nephrectomy.

Submitted 12 October 2014; Accepted 31 December 2014

\section{INTRODUCTION}

Percutaneous nephrostomy (PCN) using a fine needle and a guidewire, originally described by Gunther et al. is more than thirty years later the more widespread and accepted way to decompress the renal cavities when ureteral obstruction or anatomic conditions preclude the endoscopic placement of an ureteral double J stent (1). The reported technical success rate ranges between 98 and $100 \%$ in a review dealing with both emergency and routine PCN catheter placement (2). The risk of technical failure is slightly higher in obese patients and in absence of collecting system dilatation, where a success rate of 92 to $96 \%$ has been reported $(3,4)$. The more relevant acute major complications of this endourological procedure consist in sepsis, hemorrhage, vascular injury, bowel and pleural perforation (5). There is surprisingly very little data in the literature concerning long-term complications, mostly related to the presence of intrarenal foreign bodies as a consequence of technical or device failures during the performance of the procedure.

\section{Case report}

A 53-year-old woman was admitted for treatment of a right kidney of reduced size. Previous history revealed right pyelolithotomy for staghorn stone fourteen months earlier at a district hospital, with postoperative high temperature and early spontaneous dislodgement of the nephrostomy tube. The ureteral double J stent left at surgery was removed after 30 days. Two months later the patient presented flank pain and fever and underwent at the same hospital an unsuccessful attempt at endoscopic double J stent placement. Sonography revealed moderate dilation of the pelvicalyceal cavities, and 5 months after surgery at the same institution there was one failed attempt of percutaneous nephrostomy placement, followed by a successful one 7 days later. No detailed reports of both procedures were available in the patient medical records. However the PCN catheter was maintained for a few days and then removed.

At hospitalization in our institution sequential renal scan with 99 DTPA Tecnetium revealed a $19.6 \%$ function on the right side and $80.4 \%$ on the left. CT scan showed a kidney of reduced size with residual stone fragments and an intrarenal "device" protruding inside the renal cavities at the level of the lower pole, which was judged to be the fractured end of double J stent or nephrostomy catheter by the Radiologist who read the scan (Figures 1, 2). An open nephrectomy was performed using an anterolateral extraperitoneal approach, and extensive adhesions were found intraoperatively, particularly on the posterior 


\section{Figure 1.}

Axial CT scan of the right kidney revealing a radiopaque curved structure inside the dilated lower calyx with a wider extremity lodged inside the thinned renal parenchyma.

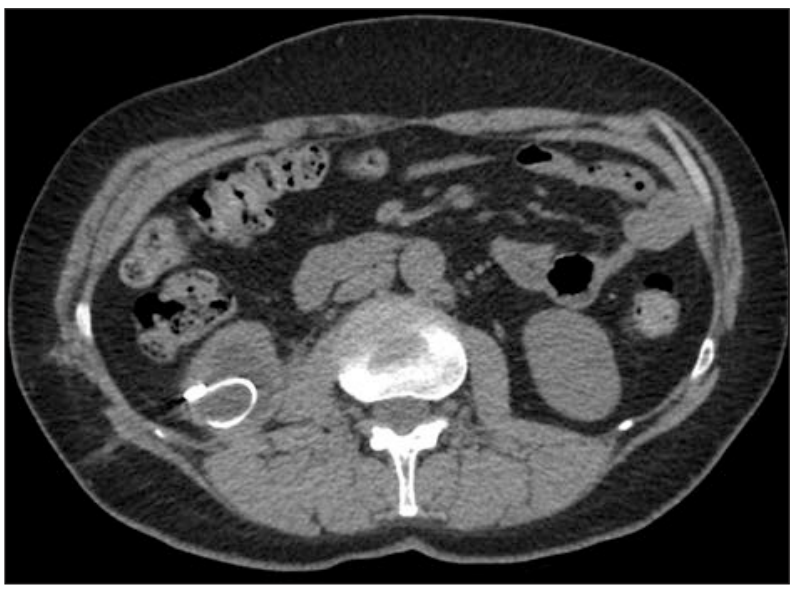

Figure 2.

Volume rendering image of the kidneys, revealing on the right side residual stones and the intrarenal "device".

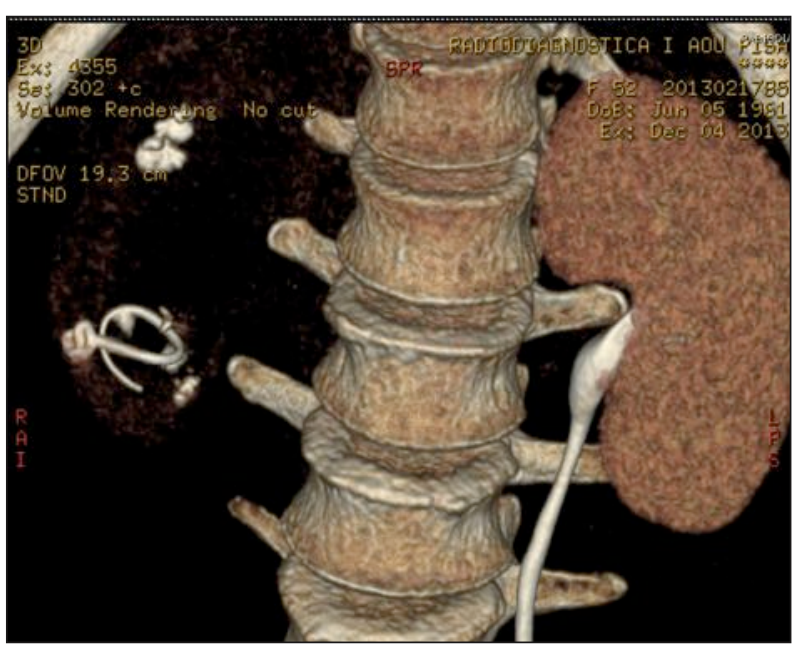

Figure 3.

Appearance of the guidewire distal extremity removed from the nephrectomy specimen, with a densely coiled midportion which was originally located inside the renal tissue of the lower pole.

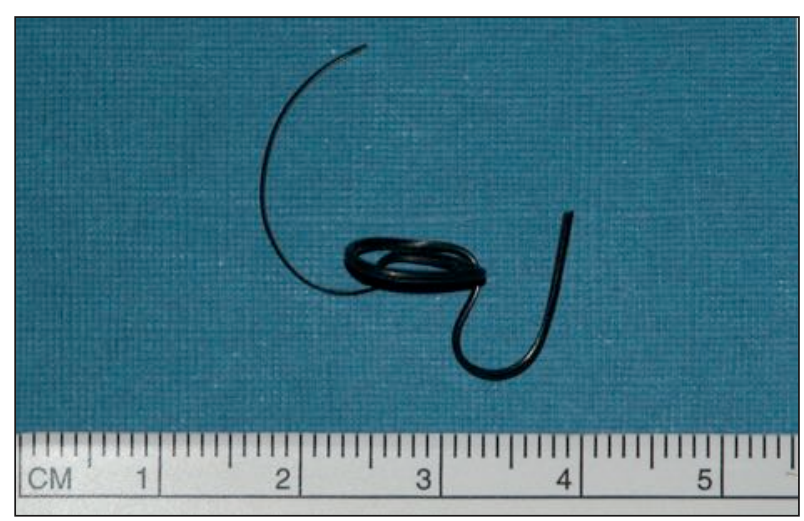

planes. Opening the operative specimen with an incision on the lateral kidney surface, it appeared that the "device" consisted in the tapered distal extremity of an hydrophilic guidewire broken during one of the previous attempts at percutaneous nephrostomy placement (Figure 3): the tightly coiled central section was wedged inside the renal tissue. Postoperative course was uneventful and the patient was discharged on the sixth postoperative day.

\section{Discussion}

To the best of our knowledge, this is the first report of a tightly coiled guidewire inside the renal tissue and the pyelocalyceal system. In a series of 21 retained renal foreign bodies there was only one inner core of a guidewire, successfully removed with a percutaneous approach, and the vast majority (15 out of 21) were ureteral stents. The fracture of a Lunderquist guidewire inside the pelvicalyceal system at the junction of the stiff portion with its flexible tip has also been described, and removed percutaneously as well as a fractured and retained cutting Acucise wire with stone formation (6-8). A possible cause of guidewire fragmentation during percutaneous renal procedures can also be the inadvertent damage produced by Holmium laser (9). Since no detailed reports of the two $\mathrm{PCN}$ catheter procedures were available in the patient medical records, we can hypothesize that during the first attempt, due to the presence of intense perirenal fibrosis, documented also at surgery, following needle puncture of the lower calyx, further advancement of the guide wire was difficult, due also to an inappropriate angle between the needle and the calyx. When withdrawal was attempted, it resulted in tight coiling of the guidewire and subsequent breakage at the junction between the stiff metallic section and the hydrophilic one. From the imaging point of view the CT scan, particularly the volume rendering, accurately depicted the unusual occurrence of a tightly coiled portion of the guidewire lodged in the thinned renal parenchyma, but the exact nature of the "device" as a guidewire was not preventively determined.

However this did not make any difference for the patient, since the kidney needed surgical removal and an endourological retrieval procedure was out of question.

\section{Conclusions}

In endourological procedures as in open surgery, a careful assessment of all equipment at the end of the procedure is required, either successful or not, especially of guidewires and laser fibers, used during all the passages to assess for damage and the risk of retained fragments in the urinary tract. A superficial check can result in the foreign body acting as a nidus for infection or stone formation or mimic a renal neoplasm (10-12).

The diagnosis and the extraction of retained renal foreign bodies can be challenging for the endourologist. Although a retrograde endoscopic approach should be tried first for foreign bodies related to previous endourological procedures, a percutaneous renal access must be considered to treat large stone burden often found on or with these retained objects: we believe in a rational approach where the treatment should be tailored to each patient (13). 


\section{References}

1. Gunter R, Alken P, Altwein JE. Percutaneous nephropyelostomy using a fine-needle puncture set. Radiology. 1979; 132:228-30.

2. Millward SF. Percutaneous nephrostomy: a practical approach. J Vasc Interv Radiol. 2000; 11:955-64.

3. Patel U, Hussain FF. Percutaneous nephrostomy of non dilated renal collecting system with fluoroscopic guidance: technique and results. Radiology. 2004; 233:226-33.

4. Maher MM, Fotheringham T, Lee MJ. Percutaneous nephrostomy. Semin Intervent Radiol. 2000; 17:329-39.

5. Ramchandani P, Cardella JF, Grassi CJ, et al. Quality improvement guidelines for percutaneous nephrostomy. J Vasc Interv Radiol. 2001; 12:1247-51.

6. Eisenberg MN, Lee KL, Stoller M. Endoscopic management of retained renal foreign bodies. Urology. 2009; 73:1189-94.

7. Bezirdjian DR, Tisnado J, Cho SR. Percutaneous retrieval of a broken Lunderquist guidewire from the pelvicalyceal system. South Med J. 1989; 82:1269-71.

8. Johnson JE, Conlin M. Calculus formation on a retained Acucise wire. Urology. 2001; 57:168.

9. Biyani CS, Powell CS. Guidewire fragmentation during Holmium:YAG laser endopyelotomy. Tech Urol 1998; 4:51-53.

10. Ahn J, Trost DW, Topham SL et al. Retained nephrostomy thread providing a nidus for atypical renal calcification. Br J Radiol. 1997; 70:309-10.

11. Singh V, Srinivastava A, Kapoor R, et al. Can the complicated forgotten indwelling ureteric stents be lethal? Int Urol Nephrol. 2005; 37:541-46.

12. Dogra PN, Tandon S, Ansari MS, et al. Suture foreign body granuloma masquerading as renal neoplasm. Int Nephrol. 2005; 37:27-29.

13. Bultitude MF, Tiptaft RC, Glass JM, et al. Management of encrusted ureteral stents impacted in upper tract. Urology. 2003; 62:622-26.

\section{Correspondence}

Francesca Manassero, MD PhD (Corresponding Author) francy_manassero@hotmail.com

Cristina Gabellieri, MD

gabelliericristina@interfree.it

Cesare Selli, MD

c.selli@med.unipi.it

Department of Translational Research, Section of Urology

Section of Urology, University of Pisa

Via Paradisa I - 56124 Pisa, Italy

Simona Ortori, MD

s.ortori@libero.it

Michela Gabelloni, MD

michelagabelloni@libero.it

Department of Translational Research, Section of Radiology 